\title{
Transitory Complete Response to the EpSSG Extracranial Rhabdoid Tumor Protocol in a White Adolescent with a Sickle Trait Harboring a Metastatic Renal Medullary Carcinoma
}

\author{
Pascal Chastagner ${ }^{*}, 1$ Romina Costanzia ${ }^{1}$, Aurélie Phulpin-Weibel ${ }^{1}$, Fanny \\ Fouyssac ${ }^{1}$, Godefroy deMiscault², Jacqueline Champigneulle ${ }^{3}$, Ludovic Mansuy ${ }^{1}$ \\ 1 Department of Pediatric Oncology, Children's Hospital, Vandoeuvre, France, ${ }^{2}$ Department of \\ Pediatric Surgery, Children's Hospital, Vandoeuvre, France, 3 Department of Histopathology, \\ Children's Hospital, Vandoeuvre, France
}

\begin{abstract}
Renal medullary carcinoma (RMC) is a very rare disease that is almost exclusive to young black patients with sickle cell trait. Most patients present with metastatic disease at diagnosis and have a grim prognosis. Histologic diagnosis of these tumors can be difficult in the presence of rhabdoid elements and loss of INI1 expression as observed in our case.

We describe the first known reported case of a white male adolescent with sickle cell trait, who was treated for a metastatic RMC with reticular pattern of rhabdoid-like cells and absence of INI1 expression, by the European Soft Tissue Sarcoma Study Group (EpSSG) protocol designed for rhabdoid tumors and who achieved a transitory complete response. Although complete remission was achieved, overall survival was not improved.
\end{abstract}

Keywords: renal tumor, rare tumors, pediatric hematology/oncology

\section{Introduction}

Renal medullary carcinoma (RMC) is a very rare and highly aggressive malignant epithelial neoplasm occurring in older children and young adults with sickle cell trait or sickle cell disease. The majority of patients have widelydisseminated disease at the time of diagnosis and most fail to respond to radiotherapy. Partial responses to systemic chemotherapy have been reported, but are rare and of short duration. Mortality approaches $100 \%$ and death usually occurs within a few months of diagnosis.

Received: August 2014; Accepted after review: August 2014; Published: September 2014

*Corresponding author: Pascal Chastagner, MD, PhD, Department of Pediatric Oncology, Children's Hospital, 11 Allée du Morvan 54511, Vandoeuvre, France.

Phone: +33 3831546 37; Fax: 0383154551

Email: p.chastagner@chu-nancy.fr
We report on the case of an adolescent with metastatic RMC who achieved a complete remission using a chemotherapy regimen designed for rhabdoid tumors.

\section{Case report}

A 16-year-old adopted male presented with transitory back pain improved by analgesic treatments. He subsequently developed gross hematuria, but the renal ultrasound was normal. One month later, he was hospitalized due to the aggravation of back and flank pain and new hematuria. A computed tomography (CT) scan revealed a heterogeneous mass arising from the left kidney and widespread metastases in the liver, adrenal glands, and lungs and extensive bone lesions. When the patient was referred to our department, he had 
lost $15 \%$ of his total body weight and he had anorexia, asthenia, and worsening pain that was being treated by opioids. He had an inflammatory syndrome and increased LDH level. The total body magnetic resonance imaging (MRI) and positron emission tomography (PET) scan revealed a left kidney tumor $(78 \times 48 \times 72 \mathrm{~mm})$ with significant retroperitoneal lymph node invasion, as well as pulmonary, liver, and adrenal glands nodules and multiple lytic bone lesions (Figure 1-4).

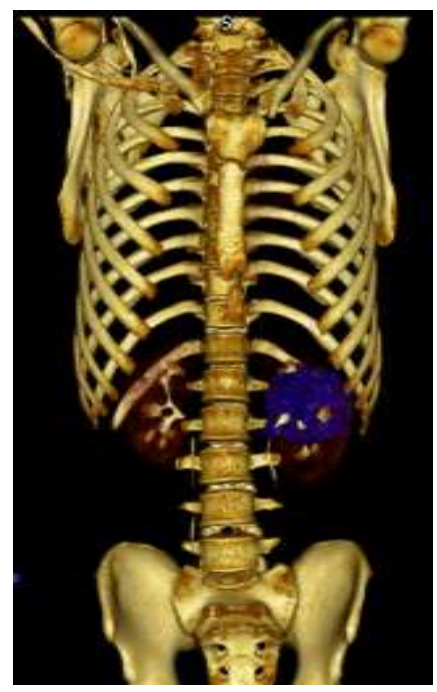

Fig. 1. 3D CT scan view of intrarenal tumor

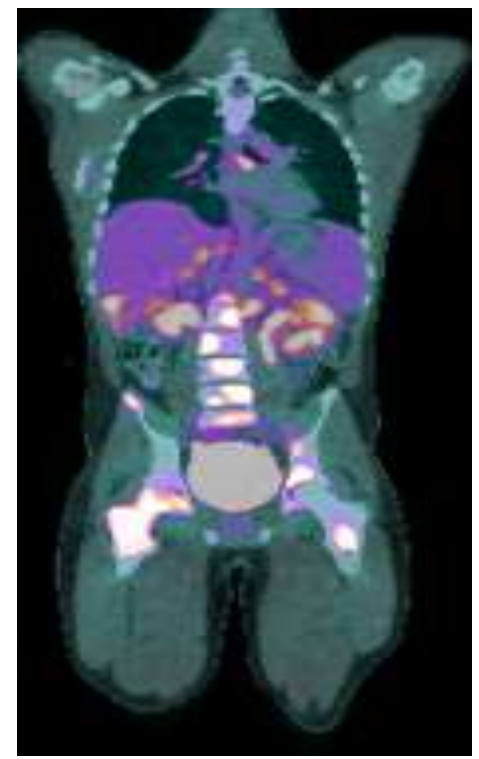

Fig. 2. Petscan: diffuse hypermetabolism of bones, liver, lymph nodes, adrenal glands

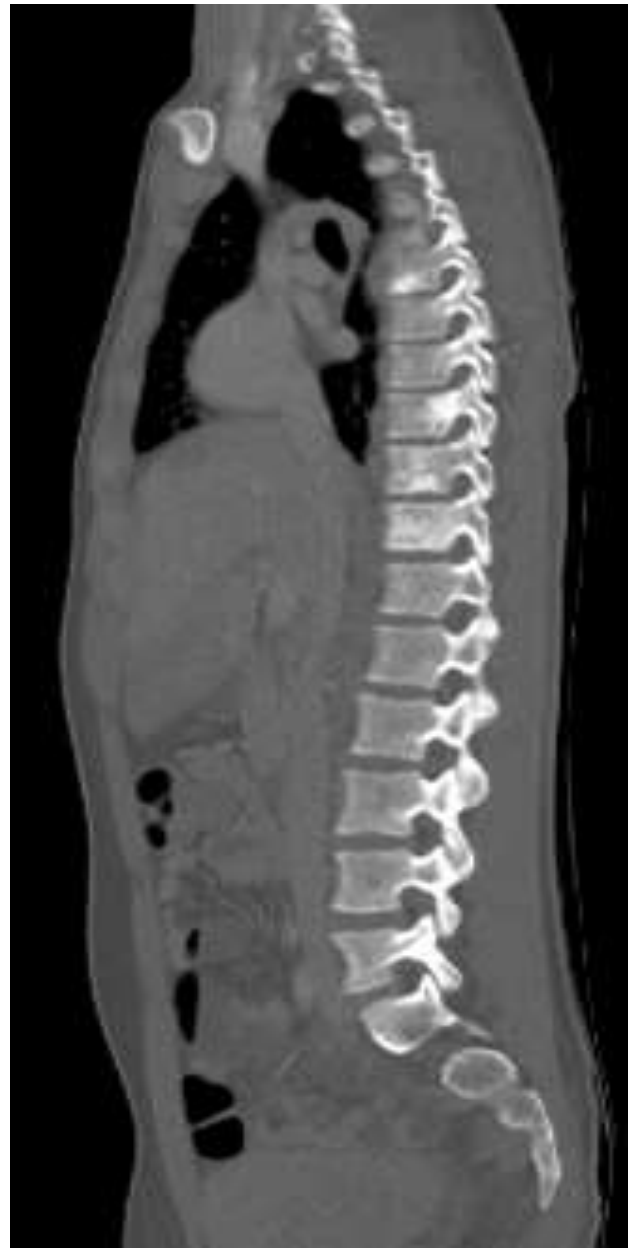

Fig. 3. MRI: Multiple spinal metastases

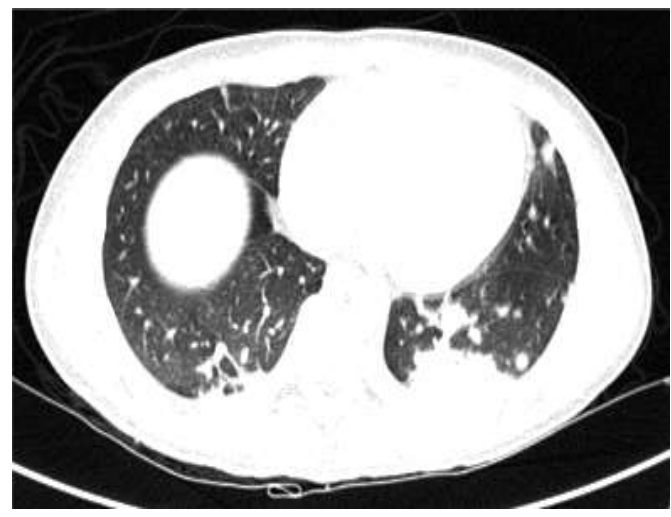

Fig. 4. CTscan: Pulmonary metastases

There was also a bone marrow invasion. Face to the very bad health condition, a fine needle biopsy was done, immediately followed by initiation of a first course of chemotherapy, prior to get the histolologic diagnosis (Figure 5 and Figure 6). 


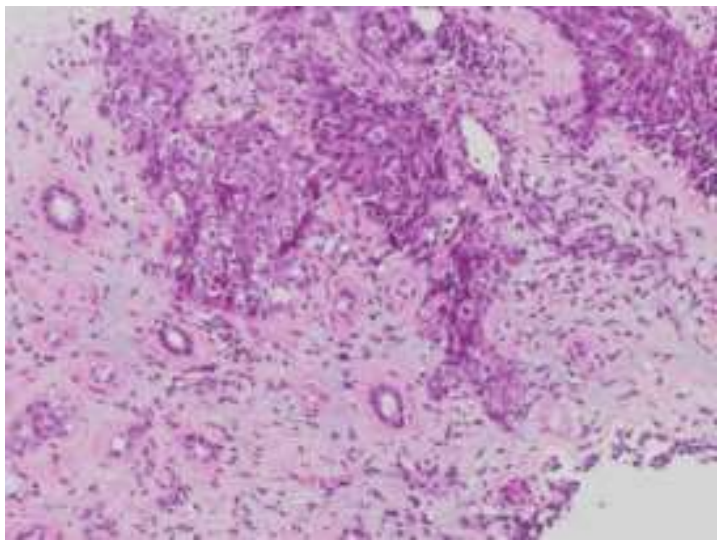

Fig. 5. Renal medulla infiltrated by tumor proliferation with epithelial aspect. Tumor cells are cohesive, medium to large sized, with reduced eosinophilic cytoplasm and large vacuolated nuclei; remnant renal tubules (lower, left) (HE, ob.x20)

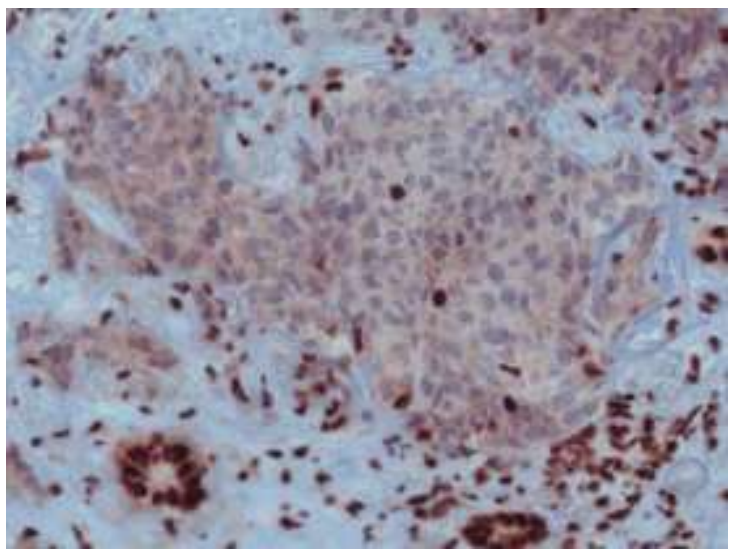

Fig. 6. Loss of expression of INI1 in tumor cells nuclei. Positive internal control: capillaries and fibroblasts. (IHC, Ab anti INI1, DAB, obx40)

Chemotherapy consisted in the European Soft Tissue Sarcoma Study Group (EpSSG) extracranial rhabdoid tumor protocol (carboplatin, vincristine, cyclophosphamide with doxorubicin, ifosfamide, and etoposide) because institutional pathologic examination concluded to a rhabdoid tumor on reticular pattern of rhabdoid-like cells with absence of INI1 expression. After 2 courses, clinical and iconographical evaluation revealed a very good partial remission of all metastatic lesions and a considerable decrease in the size of the intrarenal mass. Finally, national central review pathology concluded to RMC. In spite of the unusual Caucasian phenotype for a sickle cell disease, we performed hemoglobin electrophoresis which showed a sickle cell trait. Because of the very good response obtained with the EpSSG protocol, this treatment was continued. After 5 months of this regimen, he was in complete remission on all metastases assessed on MRI, PET scan, and bone marrow histology, and a radical left nephrectomy was made. Three months later while receiving the same chemotherapy he presented with vertebral pain. MRI and PET scan revealed a progressive disease with osteomedullary, liver, lymph nodes and pulmonary metastases. We treated him with a combination of topotecan and temozolomide, however, this resulted in a progressive disease. He received palliative cares and died 8.5 months after his initial diagnosis.

\section{Discussions}

RMC is a highly aggressive malignant epithelial neoplasm of the kidney which almost exclusively affects children and young men with a sickle cell trait or sickle cell disease and is thus known as the seventh sickle cell nephropathy [1]. The pathogenesis of this tumor is unknown. Clinically, the most common symptoms are hematuria, abdominal pain, significant weight loss, and renal mass. Most patients have extensive disease at presentation. Of the 61 patients with adequate information for staging from the 2 largest series, 11 had stage III and 50 had stage IV disease [1-3]. It is noteworthy that among frequent sites of metastases, adrenal gland is often observed, like in our case. The fatality approaches $100 \%$ within several weeks to months after diagnosis; mean survival for stage IV RMC is generally 12 weeks [1-3]. The pathologic examination makes the definitive diagnosis but rhabdoid features could be observed that may result in an incorrect diagnosis. Rhabdoid elements are occasionally seen in high-grade renal tumors, including RMC, and possibly represent a pathologic marker of aggressive behavior [4, 5]. Absence of INI1 expression, as observed in our case, is not necessarily predictive of rhabdoid histopathology, but is associated with aggressive behavior in RMC [4]. RMC and renal rhabdoid tumors share common molecular/genetic alterations which are closely 
linked to their aggressive biological behavior. In humans, the loss of INI1 expression has been reported in pediatric renal rhabdoid tumors, central nervous system (CNS) cancers, atypical teratoid/rhabdoid tumors and epithelioid sarcomas. Several genes that are over expressed in RMC, such as RNA expression of DNA topoisomerase II $\alpha$ and macrophage-stimulating 1 receptor, a tyrosine kinase, suggest the potential therapeutic utility of topoisomerase II $\alpha$ and tyrosine kinase inhibitors agents, respectively [6]. Many treatments have been proposed, but their efficacy remains poor. Partial responses were obtained on a short period using the MVAC regimen (methotrexate, vinblastine, doxorubicin, and cisplatin) [7]. Responses have also been reported using cisplatin or carboplatin in combination with gemcitabine and paclitaxel, however, death occurred 10, 12 and 24 months after the diagnosis [8]. Only two studies reported on patients in continuous remission $[5,9]$. In the reports of Watanabe et al. and Selby et al., an 8-year-old boy and a 21-year-old man treated by radical nephrectomy alone were alive 8 and 2 years after diagnosis, respectively [5, 9]. Radiation therapy efficacy is not demonstrated. New therapeutics using tyrosine kinase inhibitors and proteasome inhibitors have been recently reported. In the report of Rathmell et al., a stage IV RMC patient received imatinib without any response [7]. Another metastatic patient was treated with sunitinib maleate which did not prevent progression of the disease [7]. Ronnen et al. reported a 19-year-old boy who

\section{References}

1. Davis CJ, Jr., Mostofi FK, Sesterhenn IA. Renal medullary carcinoma. The seventh sickle cell nephropathy. Am J Surg Pathol 1995; 19:1-11.

2. Swartz MA, Karth J, Schneider DT, et al. Renal medullary carcinoma: clinical, pathologic, immunohistochemical, and genetic analysis with pathogenetic implications. Urology 2002; 60:1083-1089.

3. Guinan P, Sobin LH, Algaba F, et al. TNM staging of renal cell carcinoma: Workgroup No. 3. Union International Contre le Cancer (UICC) and the American Joint Committee on Cancer (AJCC). Cancer 1997; 80:992-993.

4. Cheng JX, Tretiakova M, Gong C, et al. Renal received 7 months of bortezomib and remained without evidence of progression after more than 27 months of follow-up [10].

In our case, we used the EpSSG pediatric rhabdoid tumor treatment protocol because the initial institutional diagnosis was a renal rhabdoid tumor. This chemotherapy allowed obtaining a complete response on metastases. It is noteworthy that no CNS relapse occurred, while this latter were reported using drugs that cannot cross the blood-brain barrier. This treatment should be considered for future cases, followed by consolidation therapy and autologous bone marrow transplantation.

This is, to our knowledge, the first case of a white patient with a sickle trait who developed a RMC. Because he was an adopted child, we did not know his family history and performed the hemoglobin electrophoresis only because of the suspicion of RMC.

\section{Conclusion}

RMC is a rare, highly aggressive tumor which primarily occurs in young black patients with sickle cell trait or disease.

Our case seems to be the first concerning a white adolescent with a sickle cell trait and confirms that rhabdoid features and absence of INI1 expression seem to be markers for clinically-aggressive tumors. While a complete response was obtained according to the treatment of rhabdoid tumors, death occurred within a year, confirming the chemosensitivity but not, at the moment, the chemocurability.

medullary carcinoma: rhabdoid features and the absence of INI1 expression as markers of aggressive behavior. Mod Pathol 2008; 21:647652.

5. Watanabe IC, Billis A, Guimaraes MS, et al. Renal medullary carcinoma: report of seven cases from Brazil. Mod Pathol 2007; 20:914920.

6. Yang XJ, Sugimura J, Tretiakova MS, et al. Gene expression profiling of renal medullary carcinoma: potential clinical relevance. Cancer 2004; 100:976-985.

7. Rathmell WK, Monk JP. High-dose-intensity MVAC for Advanced Renal Medullary 
Carcinoma: Report of Three Cases and Literature Review. Urology 2008; 72:659-663.

8. Walsh A, Kelly DR, Vaid YN, et al. Complete response to carboplatin, gemcitabine, and paclitaxel in a patient with advanced metastatic renal medullary carcinoma. Pediatr Blood Cancer 2010; 55:1217-1220.
9. Selby DM, Simon C, Foley JP, et al. Renal medullary carcinoma: can early diagnosis lead to long-term survival? J Urol 2000; 163(4):1238. 10. Ronnen EA, Kondagunta GV, Motzer RJ. Medullary renal carcinoma and response to therapy with bortezomib. J Clin Oncol 2006; 24, e14. 